system; ${ }^{3}$

- the introduction of enhanced influenza surveillance that added directed virology surveillance to the existing sentinel general practice and laboratory-based surveillance systems. ${ }^{4}$

The whole system of health protection rests on the foundation of public health surveillance. Your notifications-whether from general or specialist medical practices, laboratories, hospitals, schools or childcare centres-are vital for running, planning and improving public health programs in New South Wales. So thanks.

\section{REFERENCES}

1. NSW Department of Health. The 1998 Measles Control Campaign in NSW. NSW Public Health Bulletin 1999; 10: 89-92.

2. NSW Department of Health. The Sydney water incident: JulySeptember 1998. NSW Public Health Bulletin 1998; 8-9: 9194.

3. NSW Department of Health. Infectious diseases, NSW: October 1998. NSW Public Health Bulletin 1998; 9: 118119.

4. NSW Department of Health. Infectious diseases, NSW: April 1998. NSW Public Health Bulletin 1998; 9: 57.

\title{
INFECTIOUS DISEASES, NSW: SEPTEMBER 1999
}

\section{TRENDS}

Reports of notifiable diseases to the end of July were largely unremarkable for this time of year (Figure 1, Table 7).

\section{NSW INFLUENZA ACTIVITY UPDATE}

\section{Summary}

Influenza activity continued at a moderately high level during July and early August as reflected by both the number of laboratory diagnoses and reported clinical activity. There was a sharp increase in both forms of surveillance activity in early July. In late July and early August, influenza A activity declined while influenza B activity increased. The influenza season appears to have arrived earlier this year than in the previous few years, and at the same time of year that respiratory syncytical virus (RSV) activity usually peaks. However, influenza activity this year has not yet exceeded the peaks achieved in recent years.

\section{Clinical activity}

Rates of reported influenza-like illness have oscillated during July and early August (Figure 2). Reports were received from more than 30 general practioners (GPs) through four public health units, including approximately 3,500 consultations per week. This source of data may include illness due to causes other than influenza.

\section{Virological activity}

The laboratory reporting rate for influenza $\mathrm{A}$ decreased markedly during July and early August; however, influenza $B$ reports increased (Figure 3 ). In the second week of August, 32 cases of influenza A were reported (30 virological, 2 serological), 15 cases of influenza B (all virological) and $39 \mathrm{RSV}$. In the same week last year, there were 88 cases of influenza $A$, no cases of influenza $B$, and 120 cases of RSV. The rate of RSV isolation has been included to show that the rates of these two viruses have increased at the same time of year this season, whereas influenza A has peaked in July-August in previous years. This source of data tends to include a high proportion of hospitalised patients, particularly children, and may not accurately reflect the affect of the illness on other sections of the community.

\section{Directed virological surveillance}

Approximately 25 to 30 nasopharyngeal or throat gargle samples from patients suffering from influenza-like illness were received each week from 10 to 15 of the sentinel GPs (that is, GPs who have been specially enrolled to provide this data) during July and early August. These samples showed a similar virological pattern to the routine laboratory reports discussed previously: the influenza A isolation rate decreased from 30 per cent of samples in mid-July to zero in the second week of August, while the rate for influenza $B$ increased from seven to 21 per cent during that period. No other respiratory viruses were isolated during the period.

There are approximately 30 sentinel GPs from Central Sydney, South Eastern Sydney, Western Sydney, Wentworth, Central Coast, Hunter, Illawarra, Greater Murray and Southern Areas participating in the scheme this year.

\section{International surveillance}

Influenza activity in the southern hemisphere reported to the World Health Organization varies considerably between countries. During the first two weeks of August, Argentina continued to report influenza A activity at the level of 'widespread outbreak', while Brazil reported 'local outbreak' activity. New Zealand reported 'sporadic' activity. South Africa continues to report flu activity at the level of 'local outbreak' for both influenza A and B, and both Chile and Paraguay reported 'sporadic' activity. 


\section{FIGURE 3}

REPORTS OF SELECTED INFECTIOUS DISEASES, NSW, JANUARY 1994 TO JULY 1999, BY MONTH OF ONSET

These are preliminary data: case counts in recent months may increase because of reporting delays cases
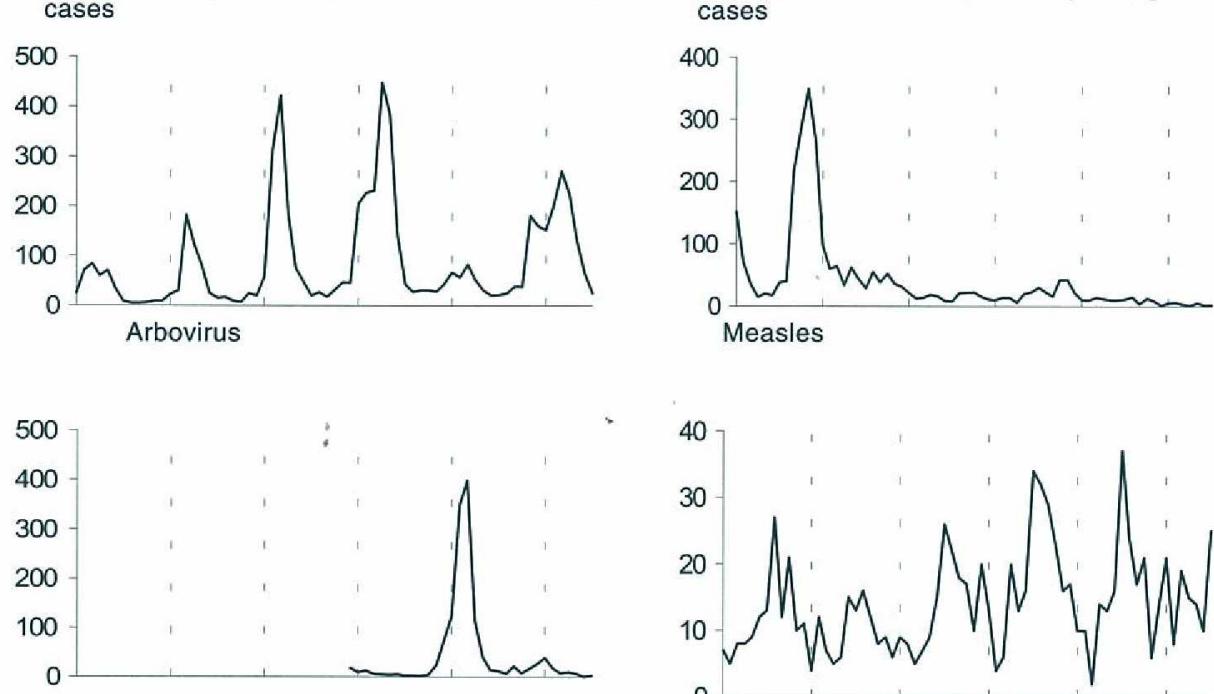

Cryptosporidiosis (not reportable before December 1996)
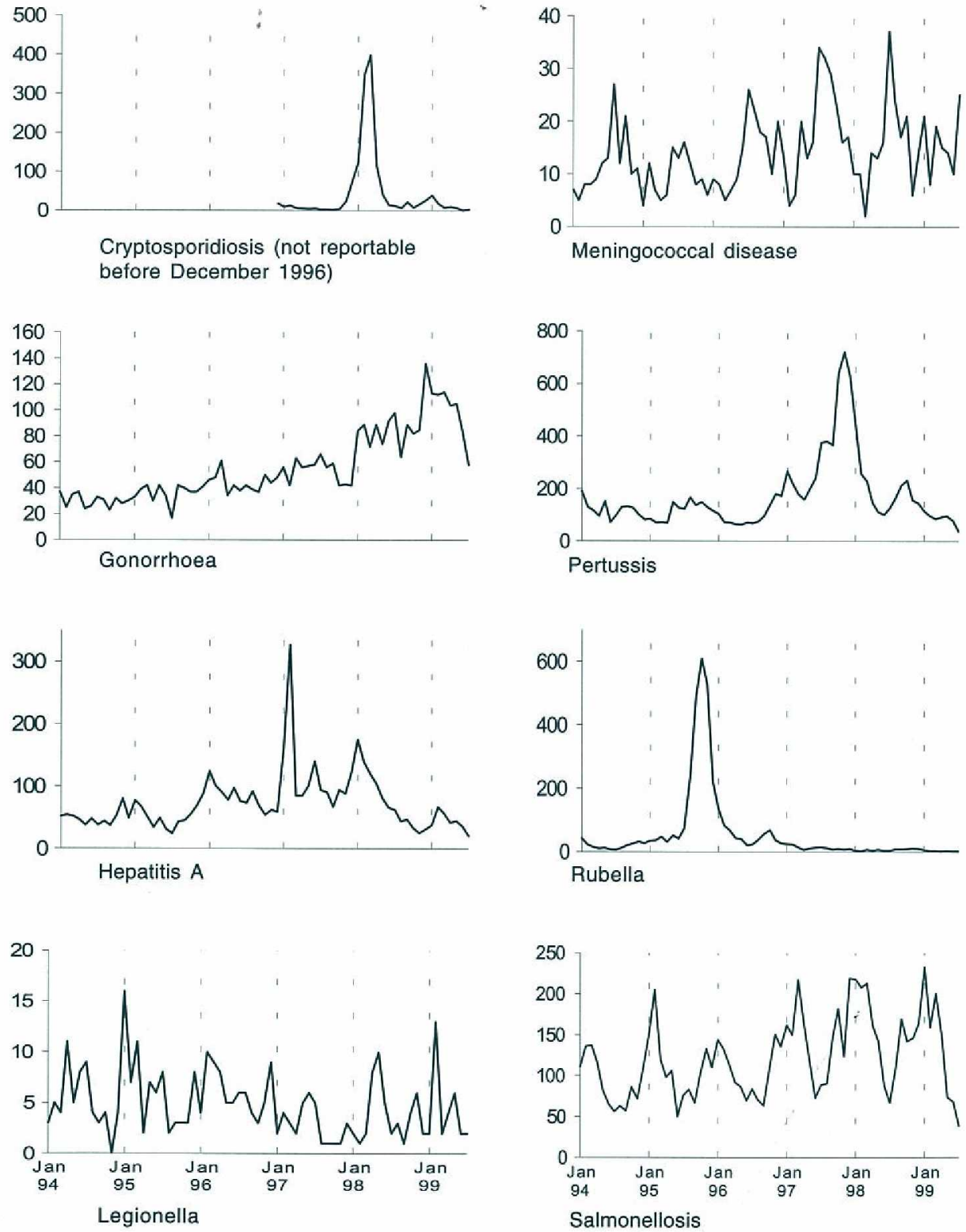

CCA HUN ILI SES NRA MNC NEA MAC MWA FWA GMA SA Total

AIV infection ${ }^{*}$

Hepatitis B: acute viral ${ }^{*}$

Hepaltis B: other"

Hepatitis C: other*

Hepatitis D: unspecified*

Hepatitis, acute viral (not otherwise specified)

Chancroid*

Chlamydia (genital)

Gonorrhoea

Vector-borne

Arboviral infection (BFV)*

Arboviral infection (RRV)

Arboviral infection (Other)

Zoonoses

Brucellosis $^{\star}$

$Q$ fever ${ }^{*}$

Respiratory and other

Legionnaires': Longbeachae

Legiannaires': Pneumophila*

Legionnaires': Other*

Leprosy

Meningococcal infection (invasive)

Mycobacterial tuberculosis

Adverse event after immunisation
$H$. influenzae b infection (invasive)

Measles

Mumps*

Pertussis

Tetanus

Faecal-oral

Botulism

Cryptosporidiosis*
Giardiasis*

Food-borne illness (not otherwise specified)

Gastroenteritis (in an institution)

Haemolytic uraemic syndrome

Hepatitis $A^{*}$

Hepatitis $E^{*}$

Listeriosis* ${ }^{*}$ (not onellosis (not otherwise specified) ${ }^{*}$

Salmonellosis (not otherwid

Typhoid and paratyphoid"
Verotoxin producing E. colit

* lab-confirmed cases only CSA $=$ Central Sydney Area NSA $=$ Northern Sydney Area $\dagger$ includes cases with unknown postcode

1
-
-
6
-
54
-
-
-
24
3
1

$\begin{array}{ll}- & 2 \\ - & 4 \\ - & - \\ 6 & 36 \\ - & - \\ - & 7 \\ - & \\ - & \\ 8 & 54 \\ 1 & 31 \\ 2 & \end{array}$

2
4
-
36
-
71
2
-
-
54
31
7

$\begin{array}{ll}- & - \\ 1 & - \\ - & -\end{array}$

$\begin{array}{llllll}- & - & - & - & - \\ - & - & - & - & - \\ - & - & - & - & -\end{array}$

$-$

$\begin{array}{rr}1 & - \\ - & - \\ - & - \\ 1 & 1 \\ - & - \\ 27 & 11 \\ - & - \\ - & - \\ - & - \\ 8 & 6 \\ 1 & 1 \\ 5 & -\end{array}$

$\begin{array}{rrrr}- & - & - & - \\ - & - & - & - \\ - & - & - & - \\ - & 1 & 6 & - \\ 3 & 31 & - & - \\ - & - & - & - \\ - & - & - & - \\ 7 & - & - & - \\ 7 & 10 & 3 & 7 \\ 2 & 2 & - & - \\ 2 & 2 & - & -\end{array}$

-
-
$\overline{5}$
-
34

for Jul† To date†

SWS $=$ South Western Sydney Area 


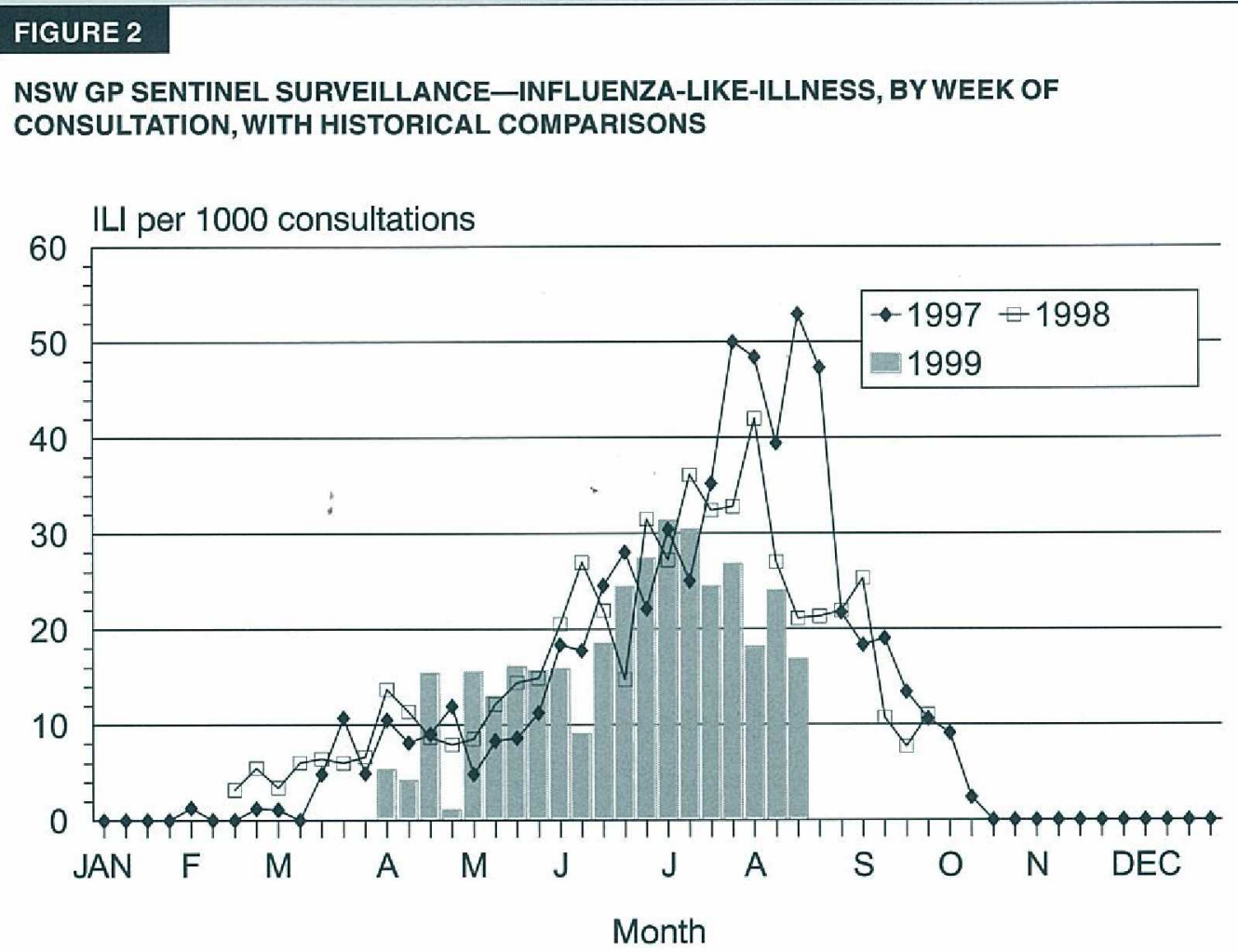

\section{FIGURE 3}

RESPIRATORY VIRUS ISOLATION RATES IN NSW, 1990-1999

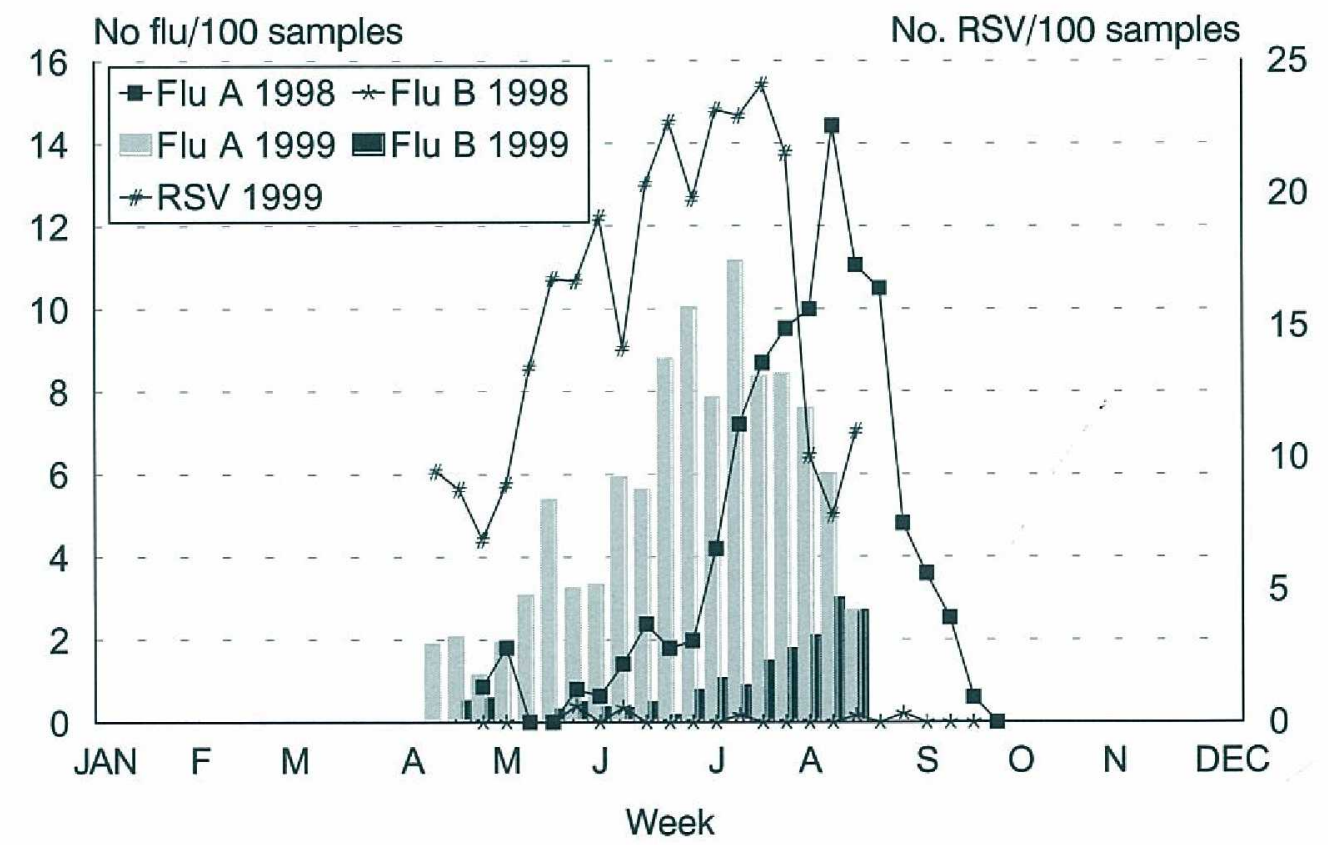

